\title{
INVESTIGATION OF POWER EFFICIENCY CHANGES IN DWDM SYSTEMS REPLACING ERBIUM-DOPED AMPLIFIERS BY SEMICONDUCTOR OPTICAL AMPLIFIERS
}

\author{
D. Pavlovs*, V. Bobrovs, M. Parfjonovs, \\ A. Alsevska, G. Ivanovs \\ Riga Technical University, \\ Institute of Telecommunications, \\ 12 Azenes Str., Riga, LV-1048, LATVIA \\ *e-mail: deniss.pavlovs_5@edu.rtu.lv
}

To evaluate potential utilization of semiconductor optical amplifiers (SOAs) as a wideband amplification alternative to erbium doped fibre amplifiers (EDFAs) in dense wavelength division multiplexed (DWDM) coherent systems, the authors discuss changes in power consumption levels required for a single bit transmission. The research evaluates the power efficiency parameter for WDM transmission systems using both amplification schemes - EDFAs that utilise standard C-band and SOAs assuming $75 \mathrm{~nm}$ amplification spectral band. The power efficiency levels have been estimated for five transmission spans with maximal distance of 640 $\mathrm{km}$. The standard $50 \mathrm{GHz}$ channel spacing has been chosen for both system configurations to allocate 100 Gbps dual-polarization quadrature phase shift keying (DP-QPSK) optical signals. The simulation schemes are described along with the critical parameters, derived from the recent relevant studies that should be taken into account considering usage of SOAs as in-line amplifiers.

Keywords: Coherent SOA-amplified transmission systems, DWDM systems, phase modulation formats, power efficiency, semiconductor optical amplifier, SOA as in-line amplifiers. 
To fulfil continuous Internet and mobile traffic demand that is dictated by a globally growing number of internet users, highdense applications, connected devices to the IP networks and machine-to-machine (M2M) connections, such as the Internet of Things (IoT) [1], several advanced techniques have been proposed in recent years to increase spectral efficiency and capacity of coherent multi-span wavelength division multiplexed (WDM) transmission systems. Among others, these include high cardinality shaped constellations, single-channel digital nonlinear compensation, and adaptive-rate capacity approaching forward error correction codes [2]-[4]. On the other hand, maximization of the spectral efficiency and capacity is tightly linked with the system energy consumption due to the necessity to maintain a specific quality of transmission (QoT) level for proper system functioning. The combination of these two factors defines a constant trade-off between efficient utilization of an available bandwidth and power required for a signal transmission through the optical fibre link, which raises ecological concerns for operators, such as carbon footprint produced by the entire sector of information and communication technologies (ICT), which is $\sim 1.4 \%$ of overall global emissions [5].

The total capacity per single-mode fibre span fundamentally is limited by the physical properties of the optical fibre, such as fibre losses, Kerr nonlinear coefficient, and dispersion coefficients, and by the optical amplifier bandwidth and noise figure (NF) parameters. This has determined the high potential of EDFA utilization as in-line optical amplifiers due to its relatively low NF and amplification bandwidth, which correlates with the lowest fibre losses within optical C-band. Nevertheless, the limited capacity of C-band cannot fulfil continuously growing traffic demand in core networks since current technologies will soon reach the limits set by the information theory and the physics of optical signal propagation through the optical fibre [6]. In the past decade, in order to extend total throughput, several solutions were designed to combine $\mathrm{C}$ and L spectral bands for WDM transmission systems. Recent studies have evaluated the C+L EDFAs [3], [7] and Raman amplification [8] providing optical bandwidth about $9 \mathrm{THz}$, but these methods still have their deficiencies, such as Raman amplifier distributed amplification and higher energy consumption. The above-mentioned traffic demand increment and existing system drawbacks have stimulated studies devoted to the examination of SOA as a more integrated and low-cost alternative. It has been demonstrated that SOA amplification bandwidth can reach up to $120 \mathrm{~nm}$ [9]. Also, the nonlinear impairments of SOAs can be reduced by different time variations of the optical signal envelope due to the present use of combined amplitude and phase modulation formats and dispersion unmanaged fibre spans. In 2017 and 2018, the specific WDM system design was demonstrated, which used custom designed in-line ultra-wideband SOAs with about $100 \mathrm{~nm}$ continuous optical amplification band [10], [11]. Additionally, the numerical investigation [12] was performed and the theoretical model [13] of SOAs as in-line optical amplifiers was developed, which provided useful guidelines for parameter definition for the current study and motivation to reconsider SOAs for inline amplification techniques within DWDM systems in terms of higher power efficiency, low costs and wide amplification range. 
The information collection and processing methodology remains similar and has been described in more detail in previous studies [14], [15]. Only input data and system parameters have been changed in accordance with the aims of the present research. Therefore, we devote more attention to parameter definition rationale. At the first step, we simulated DWDM transmission links with EDFA and SOA amplifications for several transmission lengths using the RSoft OptSim Sample-mode environment, WDM component datasheets and outcomes of recent studies with regard to specific SOA parameter definitions. The designed transmission system does not incorporate FEC (forward error correction) schemes; therefore, the main aim of this step was to reach $\mathrm{Q}$ factor level higher than $16 \mathrm{~dB}$ at a receiver, which corresponded to $10^{-9}$ bit error ratio (BER), which was measured at the most degraded channel - central channel. By reaching the required $\mathrm{Q}$ levels for a different number of fibre spans, during the next step the analytical model was created in Matlab environment for the power consumption, system capacity and power efficiency calculations, defining power consumption values from available WDM component datasheets.

To simulate optical signal propagation through the DWDM fibre link with different amplification techniques, we composed nine transmission channels spaced $(\Delta f)$ by $50 \mathrm{GHz}$ and utilising $100 \mathrm{Gbps}$ dualpolarization quadrature phase shift keying (DP-QPSK) modulation. Simplified system design is shown in Fig. 1.

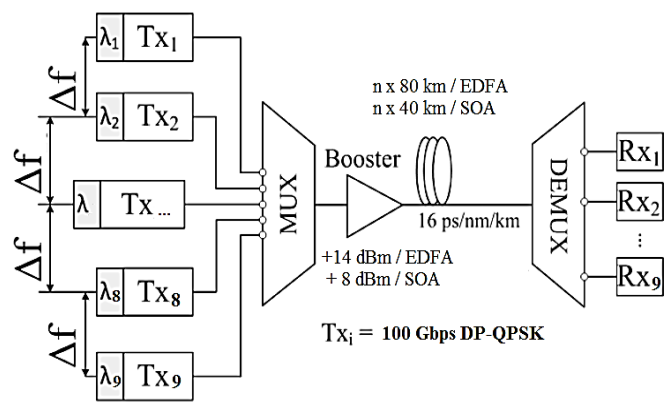

Fig. 1. Simplified DWDM system design for Q factor evaluation.

Transmitter model is displayed in Fig. 2.

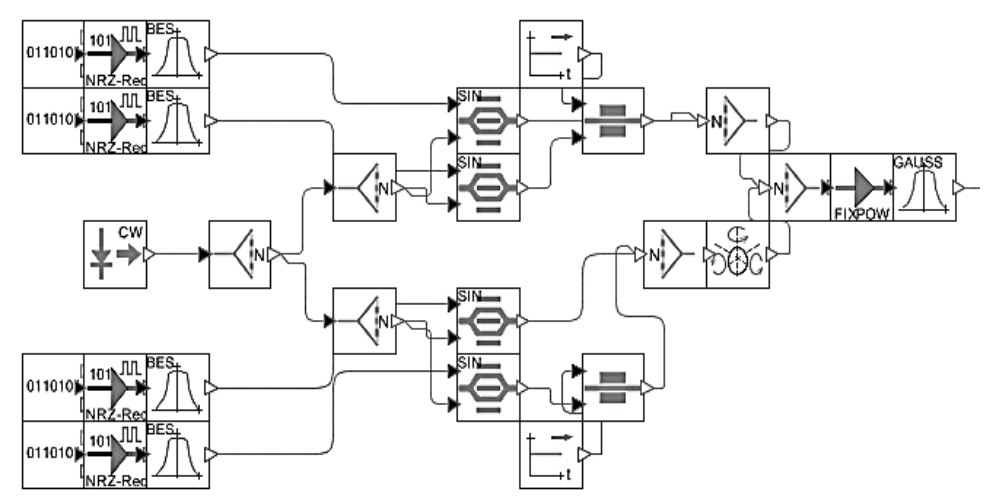

Fig. 2. 100 Gbps DP-QPSK transmitter model.

The central wavelength was set for 193.0 THz, instead of standard 193.1, in order to consider possible additional disturbances, e.g., higher fibre losses [16]. The 
continuous wave $(\mathrm{CW})$ laser source was divided into four constant $0 \mathrm{dBm}$ beams, each was managed by 25 Gbps pseudo-random bit generators through electrical pulse generators and amplitude modulators. Further, $90^{\circ}$ phase modulation was performed on two modulated optical beams and after phase modulation two signals with orthogonal phases were combined for further polarization modulation. All four optical signals were combined and transmitted through the two-sided optical Gaussian filter; $-3 \mathrm{~dB}$ bandwidth equals to $35 \mathrm{GHz}$.

The rationale for channel number was defined by the non-linear distortion statistical calculation [17], which showed that the impact of the non-linear cross-phase modulation (XPM) on specific transmission channel reached $\sim 97 \%$ of its maximum from four subsequent channels at both sides of the spectral band. The generated and combined optical signals are then amplified either by EDFA amplifier model with fixed output power of $14 \mathrm{dBm}$ and $\mathrm{NF}=4.5$, or by $\mathrm{SOA}$ model with $8 \mathrm{dBm}$ signal gain,
$N F=8$ and $14 \mathrm{dBm}$ typical saturation power $\left(P_{\text {sat }}\right)$.

From [12], it should be noted that SOA performance can have two regimes - static, assuming no gain fluctuations, and dynamic. Static regime can be found as follows (1):

$h(t)=\ln \left(G_{0}\right)-\left[\frac{P_{\text {in }}(t)}{P_{\text {sat }}}\right] *\left[e^{h(t)}-1\right]$,

where $h(t)$ is the SOA gain exponent, $G_{0}$ is the SOA small signal gain and $P_{i n}(t)$ is the SOA input power.

From (1), we can extract two different cases - linear, when $P_{i n}$ " $P_{\text {sat }}$ and non-linear, when the $P_{\text {in }}(t)$ approaches the saturation power $P_{\text {sat }}$ of SOA, which decreases the level of static gain. The degradation examples of SOA static gain exponent caused by nonlinear distortions are depicted in Fig. 3 by solving SOA gain equation using the 4th order Runge Kutta method. Two SOA saturation powers were considered to illustrate different impact degrees [12].

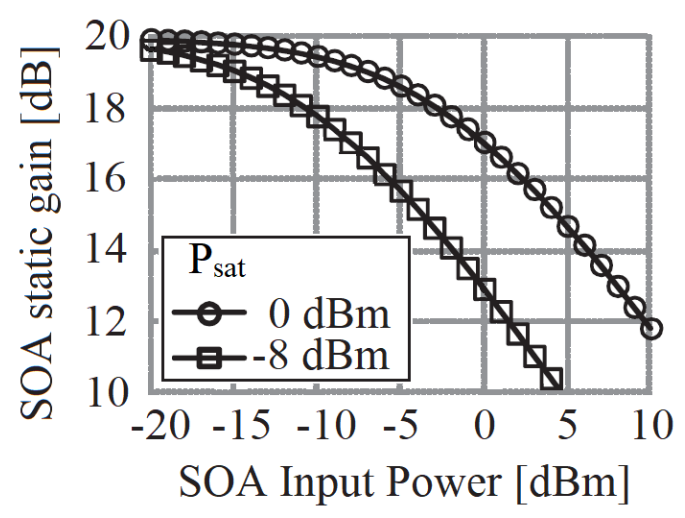

Fig. 3. SOA gain reduction caused by input power.

Dynamic regime, in its turn, presumes the $P_{i n}(t)$ and $h(t)$ oscillate around their medians $P_{i n \_a v}(2)$ and $h_{a v}(3)$ :

$P_{\text {in }}(t)=P_{\text {in_av }}+\Delta P_{\text {in }}(t)$

$$
h(t)=h_{a v}+\Delta h(t) .
$$

In the dynamic regime the SOA gain, considering few approximations [12], can be expressed as follows (4): 
$\left[1+\tau_{c} * \frac{d}{d t}\right] * \Delta h(t)=-\left[\frac{\Delta P_{\text {in }}(t)}{P_{\text {sat }}}\right] * e^{h_{a v}}$,

where $\tau_{c}$ refers to carrier lifetime. From Eq. (4), it can be concluded that the dynamic SOA gain (gain fluctuation) does not take place in the linear regime $\left(P_{i n}(t) \ll P_{s a t}\right)$. Nevertheless, it should be noted that in the nonlinear state the gain fluctuations are affected by the signal envelope oscillations in the SOA input. This raises additional considerations and points to the sensitivity of the SOA nonlinearities to modulation formats using time-varying power envelopes [12] and provides the rationale for signal modulation choice in this research. As an example, to depict correlation between input and saturation power relation and Q level, it can be calculated that if the input power takes two values of $0 \mathrm{dBm}$ and $5 \mathrm{dBm}$ with 6 $\mathrm{dBm}$ saturation power, the $Q^{2}$ level reaches $12 \mathrm{~dB}$ and $5 \mathrm{~dB}$, respectively $\left(\triangle Q^{2}=7 \mathrm{~dB}\right)$. It should be mentioned that SOAs with the higher saturation power are less exposed to the adverse non-linear distortions [12].

The above-mentioned relations determined the $40 \mathrm{~km}$ optical fibre span $(L s)$ for SOA system, since the operation around 14-16 $\mathrm{dBm}$ power for higher fibre span losses could adversely impact the spectral shape of amplification curve and, as a result, overall system performance. This scenario needs to be studied in more detail separately in future research.

Further, the amplified signal was launched into the optical fibre (using $40 \mathrm{~km}$ span for SOA systems and $80 \mathrm{~km}$ for EDFA systems), where loss $\alpha=0.2 \mathrm{~dB} / \mathrm{km}$, effective area $=80 \mu \mathrm{m} 2$, the dispersion coefficient $D=16 \mathrm{ps} / \mathrm{nm} / \mathrm{km}$, and the Kerr nonlinear coefficient $\gamma=1.261 / \mathrm{W} / \mathrm{km}$. Guided by the recent studies which evaluated signal transmission quality by considering different SOA parameters and WDM transmission system configurations [12], [13], it was decided to compensate accumulated dispersion after an opto-electric conversion by the electronic dispersion compensation (EDC) module at a receiver side instead of optical fibre dispersion compensation module (DCM) incorporated directly after the transmission fibre link.

At a receiver side, each signal was filtered from a common optical flow by twosided Gaussian filter; $-3 \mathrm{~dB}$ bandwidth was carefully tuned for each transmission length in order to define bandwidth value granting the highest transmission quality. Such evaluation example for SOA system with $L p=80$ $\mathrm{km}$ is depicted in Fig. 4.

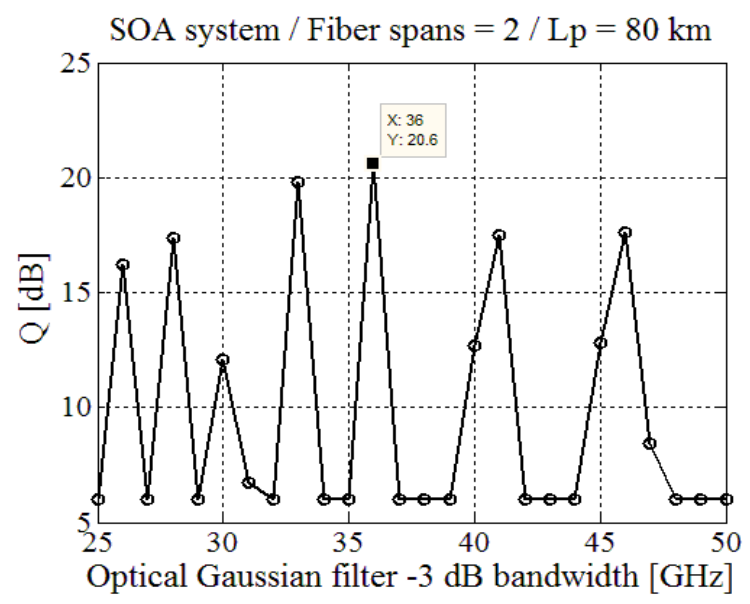

Fig. 4. Optical Gaussian filter tuning. 
Further, 100 Gbps DP-QPSK signal was divided into four $25 \mathrm{Gbps}$ signals by 4 by 4 QPSK split combiner, based on their phase and polarization and then converted by PIN photodetectors into electrical sig- nals, passed through Bessel filter, EDC and detected by $\mathrm{Q}$ estimator and electrical scope. Receiver block diagram is shown in Fig. 5.

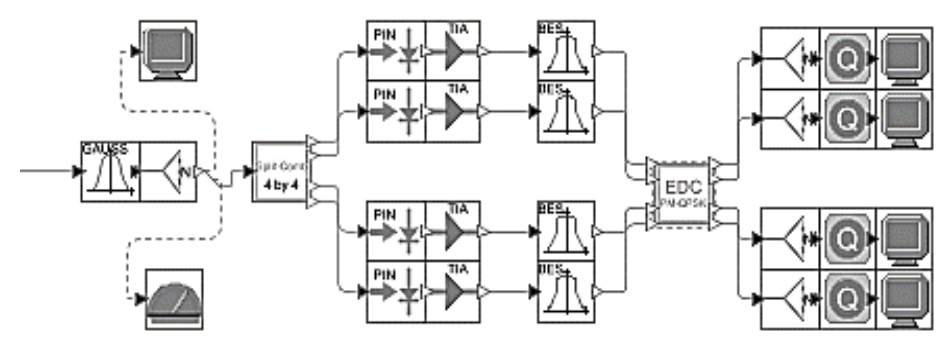

Fig. 5. 100 Gbps DP-QPSK receiver model.

The transmission length was simulated by using an iteration loop feature and specifying a number of fibre spans ( Nsp) for EDFA and SOA amplification systems. In this study, we simulated 5 transmission lengths $-80 \mathrm{~km}, 160 \mathrm{~km}, 240 \mathrm{~km}, 320 \mathrm{~km}$ and $640 \mathrm{~km}$ and for each we found a set of parameters to fulfil Q level requirement $>16$
$\mathrm{dB}$ using standard channel spacing $\Delta f=50$ GHz. It strengthened the assumption that SOAs could be used as alternative amplification devices instead of EDFAs, maintaining the same frequency grid, which in its turn could make strategic implementation more feasible.

\section{RESULTS AND DISCUSSION}

During the next step, the analytical model was designed within Matlab environment. Considering channel granularity $\Delta f=50 \mathrm{GHz}$, the utilized bandwidths were defined separately for EDFA, which was standard C-band (1530-1565 nm) with 86 channels $\left(N_{\text {ch edfa }}\right)$, and for SOA amplification systems - 1530-1605 nm, excluding wavelengths from $1565 \mathrm{~nm}$ to $1569 \mathrm{~nm}$ due to a lack of available laser sources in the transition interval between $\mathrm{C}$ and $\mathrm{L}$ bands [12], having 182 transmission channels in total amplified by SOAs $\left(N_{c h \_s o s}\right)$. Such allocation resulted in $\approx 2.12$ times greater transmission capacity (18.2 TBit/s) and $7 \%$ higher spectral efficiency compared to EDFA $(S E=2.10$ and $1.96 \mathrm{bit} / \mathrm{Hz}$, respectively).
Further, the power consumption levels were defined based on available component datasheets: for DP-QPSK transceivers $P_{t x}=$ $19 \mathrm{~W}$, SOA $P_{\text {soa amp }}=4 \mathrm{~W}$ and EDFA power

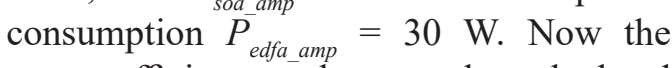
power efficiency values can be calculated and compared (5) (Figs. 6 and 7).

$P E_{x}=P_{x} / B W_{x}$,

where $X$ refers to specific parameter of either EDFA or SOA system, $P E_{x}$ - power efficiency $[\mathrm{W} / \mathrm{Hz}], B W_{x}-$ utilised spectral bandwidth [Hz], $P_{x}$ - total power consumption [W].

$P_{x}=N_{c h \_x} * P_{x}+N_{s p} * P_{x \_a m p}$. 
Unlike results of the previous studies [14], [15], [18], power consumption of ROADM terminals was not included in total power consumption calculations, focusing on system components that required defining the amount of used power and emphasising signal generation and regeneration power needs.

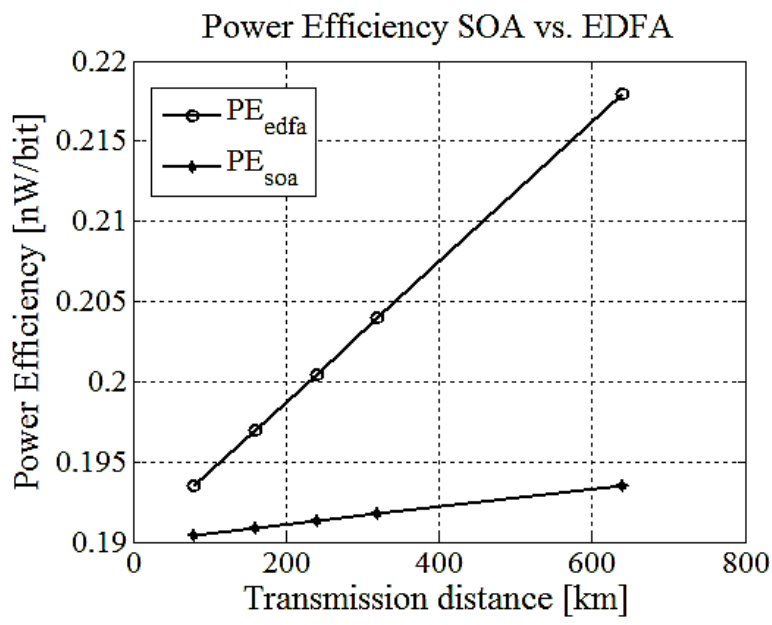

Fig. 6. Power efficiency levels at the considered transmission distances.

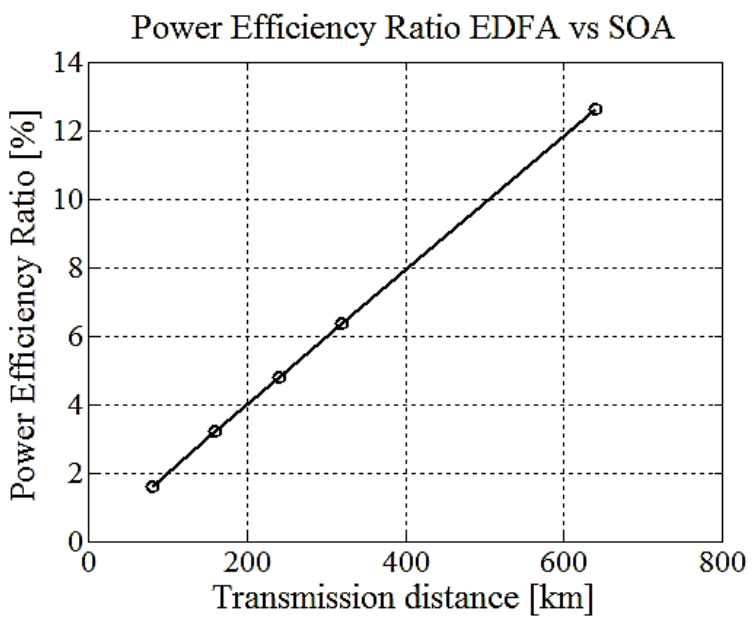

Fig. 7. Relation of power efficiency levels for EDFA and SOA amplification systems.

\section{CONCLUSION}

Motivated by a growing number of studies focused on SOA performance evaluations as an alternative low-cost ampli- fication component for DWDM systems, the main aim of this study was to evaluate the impact on the power efficiency levels 
substituting EDFAs with SOAs, considering recent findings regarding SOA capabilities and relevant analytical methods. To reach this aim, in the current research we evaluated and compared the power efficiency levels in the proposed substitution scenario, when total capacity of EDFAamplified optical link, which utilised 86 channels in C-Band, was doubled by usage of SOAs instead, providing 182 transmission channels due to wider amplification band. In both configurations, 100 Gbps DPQPSK transmission signals separated by 50 GHz channel spacing were used.

During the first simulation step, it was shown that considering correlation between non-linear distortions and ratio of semiconductor amplifier input power $P_{\text {in }}(t)$ and saturation power $P_{s a t}$, it was possible to adjust transmission parameters in such a way to guarantee $\mathrm{Q}$ factor at the receiving node greater than $16 \mathrm{~dB}$ over $640 \mathrm{~km}$ fibre link.
Further, based on the obtained results and available transmission system component parameters, the power efficiency levels were calculated. The comparison of calculated values showed that the system with SOA amplification required less energy for a single bit transmission and that difference became more considerable for greater transmission distances - from $1.6 \%$ to $12.6 \%$, for $80 \mathrm{~km}$ and $640 \mathrm{~km}$, respectively, proving that SOAs along with relatively low component costs, could grant additional ecological and financial benefits.

It should be noted that, since this is the first step to assess energy consumption volumes for SOA amplified systems, several potentially critical system parameters, physical impairments and hardware availability and compatibility questions are out of the scope of this research and must be analysed in future studies.

\section{REFERENCES}

1. Cisco Systems, Inc. (2020). Cisco Annual Internet Report (2018-2023). Available at https://www.cisco.com/c/en/us/solutions/ collateral/executive-perspectives/annualinternet-report/white-paper-c11-741490.html

2. Ghazisaeidi, A., de Jauregui Ruiz, I.F., Rios-Muller, R., Schmalen, L., Tran, P., Brindel, P., ...\& Renaudier, J. (2016). $65 \mathrm{~Tb} / \mathrm{s}$ transoceanic transmission using probabilistically shaped PDM-64QAM. In: 42nd European Conference on Optical Communication, 18-22 September 2016, Dusseldorf, Germany.

3. Cai, J.-X., Batshon, H.G., Mazurczyk, M.V., Sinkin, O.V., Wang, D., Paskov, M., .. \& F Foursa, D.G. (2018). 51.5 $\mathrm{Tb} / \mathrm{s}$ Capacity over $17,107 \mathrm{~km}$ in $\mathrm{C}+\mathrm{L}$ Bandwidth Using Single-Mode Fibres and Nonlinearity Compensation. Journal of Lightwave Technology, 36 (11), 2135-2141. DOI: $10.1109 /$ JLT.2018.2802322
4. Zhang, S., Yaman, F., Huang, Y.-K., Downie, J.D., Zou, D., Wood, W.A., ... \& Inada, Y. (2016). Capacity-approaching transmission over $6375 \mathrm{~km}$ at spectral efficiency of $8.3 \mathrm{bit} / \mathrm{s} / \mathrm{Hz}$. In: 2016 Optical Fibre Communications Conference and Exhibition (OFC), 20-24 March 2016, Anaheim, CA, USA.

5. Malmodin, J., \& Lundén, D. (2018). The Energy and Carbon Footprint of the Global ICT and E\&M Sectors 2010-2015, Sustainability. Available at https://www. researchgate.net/publication/327248403 The Energy and Carbon Footprint of the Global_ICT_and_EM_Sectors_20102015

6. Essiambre, R., Kramer, G., Winzer, P.J., Foschini, G.J., \& Goebel, B. (2010). Capacity Limits of Optical Fibre Networks. Journal of Lightwave Technology, 28 (4), 662-701. DOI: 10.1109/JLT.2009.2039464 
7. Ghazisaeidi, A., de Jauregui Ruiz, I.F., RiosMuller, R., Schmalen, L., Tran, P., Brindel, P., .. \& Renaudier, J. (2017). Advanced C+L-Band Transoceanic Transmission Systems Based on Probabilistically Shaped PDM-64QAM, Journal of Lightwave Technology, 35 (7), 1291-1299. DOI: 10.1109/JLT.2017.2657329

8. Ionescu, M., Lavery, D., Edwards, A., Sillekens, E., Galdino, L., Semrau, D., ... \& Bayvel, P. (2019). $74.38 \mathrm{~Tb} / \mathrm{s}$ transmission over $6300 \mathrm{~km}$ single mode fibre with hybrid EDFA/Raman amplifiers. In: Optical Fibre Communications Conference and Exhibition (OFC), (pp. 1-3), 3-7 March 2019, San Diego, California, USA. Available at https:// arxiv.org/abs/1902.09821

9. Akiyama, T., Ekawa, M., Sugawara, M., Sudo, H., Kawaguchi, K., Kuramata, A., ... \& Arakawa, Y. (2004). An ultrawide-band $(120 \mathrm{~nm})$ semiconductor optical amplifier having an extremely-high penalty-free output power of $23 \mathrm{dBm}$ realized with quantum-dot active layersb. In: Optical Fibre Communication Conference, 22 February 2004, Los Angeles, California, USA.

10. Renaudier, J., Meseguer, A.C., Ghazisaeidi, A., Tran, P., Rios-Muller, R., Brenot, R., .. \& Charlet, G. (2017). First 100$\mathrm{nm}$ continuous-band WDM transmission system with $115 \mathrm{~Tb} / \mathrm{s}$ transport over $100 \mathrm{~km}$ using novel ultra-wideband semiconductor optical amplifiers. In: 2017 European Conference on Optical Communication (ECOC), (pp. 1-3), 17-21 September 2017, Gothenburg: IEEE. DOI: 10.1109/ ECOC.2017.8346084

11. Renaudier, J., Meseguer, A.C., Ghazisaeidi, A., Brindel, P., Tran, P., Verdier, A., ... \& Charlet, G. (2018). Field trial of $100 \mathrm{~nm}$ ultra-wideband optical transport with 42GBd 16QAM real-time and 64GBd PCS64QAM channels. In 2018 European Conference on Optical Communication (ECOC), (pp. 1-3), 23-27 September 2018, Rome, Italy: IEEE. DOI: 10.1109/ ECOC.2018.8535349
12. Bendimerad, D.F., \& Frignac, Y. (2017). Numerical Investigation of SOA Nonlinear Impairments for Coherent Transmission Systems Based on SOA Amplification. Journal of Lightwave Technology, 35 (24), 5286-5295. DOI: 10.1109/ JLT.2017.2772223

13. Ghazisaeidi, A. (2019). Theory of Coherent WDM Systems Using In-line Semiconductor Optical Amplifiers. $J$. Lightwave Technol., 37, 4188-4200. DOI: 10.1109/JLT.2019.2921864

14. Pavlovs, D., Bobrovs, V., Vilcāne, K., \& Ivanovs, G. (2019). Investigation of optical signal regeneration impact on power efficiency of single-line-rate and mixedline-rate wavelength division multiplexing systems. In: PhotonIcs \& Electromagnetics Research Symposium - Spring (PIERSSpring), (pp. 896-901), 17-20 June 2019, Rome, Italy: IEEE. DOI: 10.1109/PIERSSpring46901.2019.9017644

15. Pavlovs, D., Bobrovs, V., Parfjonovs, M., Alsevska, A., \& Ivanovs, G. (2017). Evaluation of Signal Regeneration Impact on the Power Efficiency of Long-Haul DWDM Systems. Latvian Journal of Physics and Technical Sciences, 54 (5), $68-$ 77. DOI: 10.1515/lpts-2017-0035

16. Agrawal, G.P. (2007). Nonlinear Fibre Optics. New York, NY, USA: Academic.

17. Velasco, L., Jirattigalachote, A., Ruiz, M., Monti, P., Wosinska, L., \& Junyent, G. (2012). Statistical approach for fast impairment-aware provisioning in dynamic all-optical networks. IEEE/OSA Journal of Optical Communications and Networking, 4 (2), 130-141. DOI: 10.1364/ JOCN.4.000130

18. Pavlovs, D., Parts, R., Muratbeck, D., \& Bobrovs, V. (2017). Comparison of power efficiency and signal regeneration impact in the SLR DWDM transmission systems with different spectral band. In: Progress in Electromagnetics Research Symposium Fall (PIERS - FALL), (pp. 1122-1127), 1922 November 2017. Singapore: IEEE. 